Please quote as: Dünnebeil, S.; Leimeister, J. M. \& Krcmar, H. (2011): Marketplaces for health applications. Assessment of Requirements in Case of the German Public Health System. In: 4. International Joint Conference on Biomedical Engineering Systems and Technologies (BIOSTEC) 2011, Rome, Italy. 


\title{
MARKETPLACES FOR HEALTH APPLICATIONS Assessment of Requirements in Case of the German Public Health System
}

\author{
Sebastian Dünnebeil ${ }^{1}$, Jan Marco Leimeister ${ }^{2}$, Helmut Krcmar $^{1}$ \\ ${ }^{1}$ Department of Informatics, Technische Universität München, Germany \\ duennebe@in.tum.de, krcmar@in.tum.de \\ ${ }^{2}$ Department of Economics, Universität Kassel, Germany \\ leimeister@uni-kassel.de
}

\begin{abstract}
Keywords: $\quad$ Application Store, Healthcare, e-health, Telemedicine, Public Health System, Treatment Quality, Treatment Cost, Marketplace, SaaS

Abstract: $\quad$ Multiple innovations in e-health have the proven potential to improve treatment success, reduce adverse events and healthcare spending. Despite the promising potential, verified in various studies, the diffusion speed of healthcare application is very low. A major challenge in healthcare is therefore not only the research and development for improved treatment, but also the comprehensive and effective diffusion of selected technologies to caregivers and patients. Hence, the paper investigates the theoretical background influencing the diffusion of innovations. It adopts the concept of application stores, which have shown high potential as accelerators for extensive distribution of software applications, for the domain of e-health. Requirements for such a marketplace are derived from the constraints of the public health system. The involved actors are identified and linked in a high level model.
\end{abstract}

\section{INTRODUCTION}

Health systems around the world have seen multiple innovations in the field of electronic health (e-health). The benefits of these applications are proven in many cases; however, the diffusion speed of such applications is currently very low (European Comission, 2007). Telemonitoring e.g. has strong evidence to be beneficial in many aspects of healthcare. Hospitalization, mortality rate, lack of work, and the overall treatment costs can be reduced partially tremendously by telemonitoring, up to $40 \%$ (Helms et al., 2007, Kempf and Schulz, 2008); however, the software based prevention method is not commonly used yet. Also medical errors, which are called adverse event and cause more death than breast cancer, traffic accidents, and AIDS combined, can be limited by the usage of e-health application. Hence utilization of such supporting information systems (IS) is urgently recommended by the European Authorities (European Comission, 2007). Applications managing the accurate discharge of prescription show significant advantages, compared to the traditional handling. These are able to decrease the rate in unadjusted absolute death rate by $27 \%$ for cardio-vascular patients after one year (Lappé et al., 2004). Considering the fact that wrong medication in the united States cost \$4.4 Billion in 2006 (IOM, 2006) and 37.4\% of all adverse events are caused by wrong medication (Aranaz-Andrés et al., 2008), the diffusion of such technologies should be the subject of efforts across the whole society. As the technology is theoretically available, the benefits are proven; a method to organize and accelerate the distribution is an important factor. Therefore the paper investigates the reasons for slow adoption of e-health and suggests a model to improve the situation.

\subsection{Diffusion of Innovations}

According to Rogers (Rogers, 1995) the diffusion speed of innovations depends on five factors (figure 1). These factors are assumed to be 
valid for healthcare innovations; they are therefore applied to the area of e-health applications. Goal of this step is to explain the unsatisfying situation, visible by now.

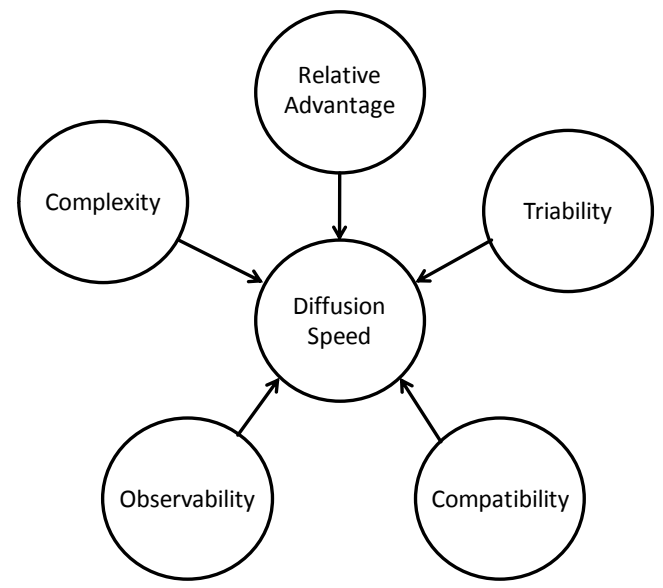

Figure 1 - Diffusion speed of innovations

\section{Relative Advantage}

If the new treatment method can achieve a relative avantage compared to the current handling, it fosters the underlying innovation. Main stakeholders involved in the treatment are caregivers, patients, and the founders (normally health insurances or tax payers). The examples above show that the relative advantage is obvious, at least in the case of patients and founders, as treatment success improves and treatment cost drops. The usage condition must therefore be changed in a way that caregivers also benefit from the relative advantage when using ehealth applications. This could be achieved by financial incentives. Cases where expected efficiency advantages resulting from the application of health information technology (IT) are not, at least partially, distributed to the caregivers are likely to gain little acceptance within this group. In the case of the German health card this problem caused a tremendous cut back in functions offered by the system, due to the resistance of the caregivers (Tuffs, 2008). No insentive structures were planned for this group, the reduction of overall treatment spendings, resulting from better health care, raised the fear of lower income for phyiscians without adequate compensation (Bernnat, 2006).

\section{Complexity}

Innovations must be easy to handle for the involved actors. Considering the fact that a an average physician-patient contact only lasts about seven minutes (Kurt, 2001), complex applications are difficult to handle for either side. Handling for the patients must be especially easy, as they have high variation in cognitive reaction and technical background. Caregivers genarally do see themselves, at least partially, responsible for the education and support of their patients, when they use e-health services. (Dünnebeil et al., 2010b) This raises a high scepticism concerning the extra effort caused by the support of patients and requires a comprehensive program in order to distribute the education work to various institutions.

\section{Compatibility}

Applications must be integrated into the application landscape of the software that is already deployed in the health institutions. The exchange of data with existing applications is essential in many cases. Missing data exchange leads to the usage of multible software systems during the treatment of one patient. In case of multimorbidity several treatment processes have to be combined. Hence software integration should result in one interface instead of multible. In extreme cases the divertion into seperated systems can even cause patient's death, caused by cognitive overload of caregivers (Zuehlke et al., 2010). Compatibility can be partially ensured by interoperability of distinct IS. Several approaches exist to ensure syntactic and semantic interoperability (Pedersen and Hasselbring, 2004). However, the compatibility is not fully insured for combinations of various software modules yet.

\section{Triability}

Potential users must be able to try e-health applications before their adoption decision. The case of the German health card system, which tried to establish mandatory software usage for e.g. prescribing, has shown that voluntary usage has advantages. Several systems should compete in terms of user friendliness. The chance to try different applications for the same purpose gives the possibility to adopt the system which matches the users' individual requirements best.

\section{Observability}

Little of the advantages of e.g. telemonitoring or prescription applications are known to the public by now. If patients can observe that other patients, who do use e-health applications personally or who's caregivers are supported by such software, receive a better treatment, they will likely request the services during medical treatment, too. Same counts for caregivers who can observe advantages in terms of earnings or quality achieved by colleagues using ehealth applications. 


\section{APPLICATION STORES}

Marketplaces for applications have the potential to deliver software from various developers distributed all over the world to users. While mobile phones had few applications installed in the past, by today a mobile phone can host any combination of up to 225,000 applications (Holzer and Ondrus, 2009). The revenue created with the App Store of apple currently exceeds $\$ 1$ Billion; more than 5 Billon applications were downloaded and deployed on the devices of the company so far. Considering the total sales of the iPhone and the iPod Touch, which reached 34 Million units by 2009, an average deployment of about 50 applications on one device can be derived for the year 2009 (AppleInsider, 2010). This turns a modern Smartphone into a highly customizable device, which can be adapted to fulfil the user's individual requirements. The criteria for diffusion of innovations, which were discussed earlier, can be easily observed in the case of the iPhone and the App Store. The relative advantage for customers is the availability of a huge number of functionalities, offered for their device. It can therewith be customized to a degree far beyond a standardized product, as the development of functionalities does not originate in a single development entity. Also a mass customization, as in case of the car industry, cannot reach such a diversification as a huge distributed community of developers. Financial advantages are possible for both, developers, who can reach a broad audience with their software, and for the distributing company, which can earn a share of the revenue and sells the pool of functionalities available for their devices as a competitive advantage. The complexity was also reduced, as a single installation method allows the utilization of specialized applications. A modern Smartphone is no longer a device for a small group of people with high technical expertise; rather it is usable for a broad spec of users. Compatibility with e.g. common mail accounts, wireless access points and the synchronization with home computers allow the usage of personal data on various devices and in several applications. The triability has increased as the technology is nearly ubiquitously offered; users can download free evaluation versions of applications and evaluated them prior to the adoption decision. Acquaintances and retailers offer potential adopters the chance to get an inside, before the individual decision whether to adopt the technology or not is taken. The advantages of applications distributed in this way are also observable for the public by now. Many people use the applications and advertisement tries to transport the advantages to a broad target audience.
As e-health innovations can achieve advantages for patients and reduce the public healthcare expenses for chronic diseases, health authorities should be keen pushing the deployment of these technologies. If the potentials of application stores for mobile phones can be transferred to the healthcare sector, a broader distribution might be a possible result. The paper will therefore investigate if the concept of marketplaces for applications, as described for mobile communication, can be transferred to the healthcare sector. The concept turns requirements and constraints into a high level model, which can be implemented afterwards.

\section{REQUIREMENTS IN HEALTHCARE}

Medical IS are currently mostly available in fixed versions with few optional extension modules. Some Personal Health Records (PHR), as HealthVault of Microsoft, offer a data pool for patients, which can be extended and used by additional applications as heart rate watches or blood pressure monitors (Microsoft, 2007). The security issues, HealthVault is not conform to the Health Insurance Portability and Accountability Act (HIPAA) and the German privacy protection commissioner discourage patients from usage (Sunyaev et al., 2010), turn the usage of patient centred commercial health records into an unlikely approach to overcome the described diffusion problem. Especially since caregivers harshly reject private companies to act as health application providers and PHR operators (Dünnebeil et al., 2010b), other methods must be evaluated to encourage the distribution of e-health innovations.

The healthcare sector is not considered a normal market. Only less than one third of all services provided in healthcare are paid by the consumer of these services directly. Most expenses are covered by health insurances or authorities, financed by tax money. Those must not take adoption decisions based on subjective opinion; they must rather establish a traceable method to decide whether an application should be financed. A patient cantered approach is therefore not sufficient. A method to bill services to health insurances must be included. Accordingly the approach used in the mobile phone market is adopted and extended for healthcare in the following section. When designing a marketplace for e-health applications, several conditions have to be fulfilled. The paper constructs a marketplace for 
healthcare applications, considering the requirements and constraints set in the German public health system. The model is developed stepby-step, starting with the software deployment model, which is currently used for software distribution in the German public health system to a model, which includes all necessary aspects covered in this work. Goal of software development on the platform is to allow developers of medical software and telemedicine applications to reach as many users as possible. The best innovations ought to be used widely in healthcare, as they have positive medical impact.

As an example for an e-health application we look at an application for monitoring cardiac insufficiency. A software developer has an innovative concept for telemonitoring, which he wants to sell to caregivers in ambulatory care. The company is currently too small to approach a bigger number of caregivers directly. Additionally the caregivers show little interest, since they cannot bill the telemonitoring to the health insurance. Patients with a valid diagnosis for this chronic disease are able record their blood pressure and weight once a day with the system. The data from the blood pressure meter and the scale is transferred and recorded on the server of the software provider. The data transfer from the devices to the server can be automated or uploaded manually. Once the parameters run out of normal range, the physician receives a notification. The doctor's medical IS sends a request the service to see whether some of the patients he monitors have vital parameters showing impairment as indication for an infarct. Action can be taken before the patient calls in because of the symptoms. This normally saves valuable time. According to the study the saving for one patient can be expected to be as high as 6000 Euros in one year (Helms et al., 2007). A part of the savings should go to the application provider and the caregiver who performs additional extra work when using the telemonitoring application. A further share of the savings can be granted to patients with good compliance.

\subsection{Direct Distribution Model}

Traditionally software is developed as a personalized solution for one customer or released as a product, which can be sold to multiple users afterwards. Developers of the software can be either internal IT departments or self-contained software companies. The distribution channel mainly leads from the developer to the user directly, reflects therefore a 1:1 relation (figure 2). Mostly a sales force is needed to distribute software this way. Especially for non consumer products, as health IS or e-health applications, traditional retailers are not suitable.

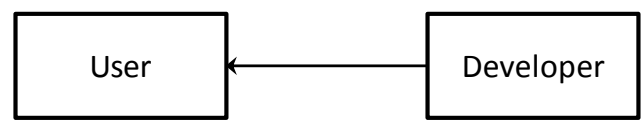

Figure 2: Traditional Software development and distribution model

Development of customized software for normal sized medical institutions is often not possible due to the size. The average institution in German ambulatory care counts about 2.1 physicians, with 86 patients visiting the practise per day (Dünnebeil et al., 2010a). With 289k Euro average annual revenue (Kassenärztliche Bundesvereinigung, 2008) the development of a customized solution is hardly affordable and not cost-effective. Most physicians in ambulatory care are therefore using standardized stand alone systems for their treatment support. The market of German health IS for ambulatory care is highly fragmented. In 2009116.895 medical IS were installed in physicians' practices. In total more than 200 different systems are deployed in primary care (KVB, 2009). For these solutions the availability of functions or modules depends on the developments of the product owner. The utilization of services is obviously also coupled with the product life cycle, as updates delivers new functions. For smaller companies this approach raises the barrier for market entry. They depend on the owners of the medical IS to include and sell the application bundled with their software. An individual market entry with standalone solutions is difficult, taking into account that nearly 170,000 physicians are practicing in German ambulatory care. Small companies can neither provide a sales force to approach a big number of physicians, nor can they provide the overall functionality of a self contained-system.

\subsection{Application Platforms for many developers}

An alternative to the one-to-one distribution of software is a marketplace for applications, accessible to many developers as a service (figure 3). This approach became hugely famous when Apple released its application marketplace called "App Store" for their devices. Apple did not intend to develop the whole software set deployable on their 
embedded devices any longer within their own company. The devices were opened for developers around the world to sell or donate their applications via the application store. The operating system running on such devices is usually not an open platform. Installation of additional software is only possible via the provider's proprietary internet platform. All applications are checked on conformity with the company's guidelines pervious to their release, especially concerning compatibility and content. The services provided by the platform are financed by a certain share of the retail price, which is retained by the store provider. The financial transactions are, at least in the case of the App Store, carried out by the provider of the store.

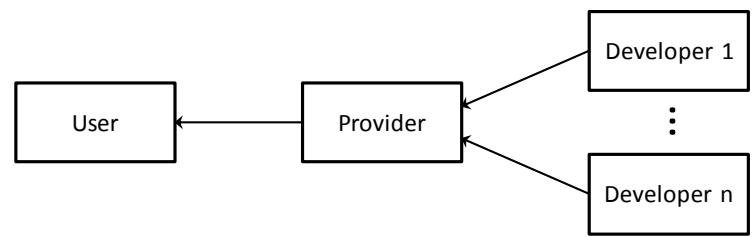

Figure 3: SW-Distribution model via a centralized provider

The resulting model is a 1-to-n distribution model, as all the devices running the applications are of one kind. This approach can be adopted and applied for the owners of the medical IS. They can offer developer platforms to enrich their software with external content. This concept, similar to the one provided by apple, is hardly feasible, considering that 200 different providers offer platforms in ambulatory care. If a developer wants to reach all patients, theoretically 200 different versions have to be provided, which requires a high administrative and technical effort. The providers of medical IS would have to agree on a common extension model, ensuring universal compatibility.

\subsection{Application Platforms for various user groups}

In case of the German health system a high fragmentation of target platforms is one constraint that has to be taken into account as there is currently no overall extension model. Most health IS in hospital or practices are deployed on open platforms as a personal computer (PC) or an application server. Software which is offered by the provider can be deployed directly to the target platforms. However the compatibility with the main medical IS has to be ensured.

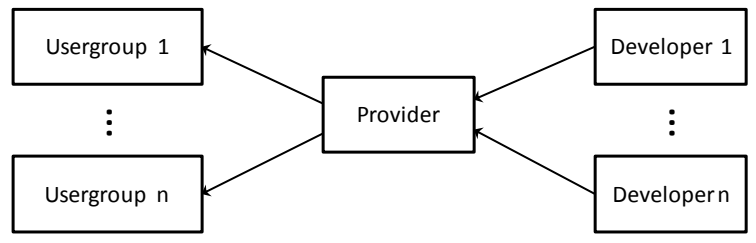

Figure 4: Distribution model with multiple developers and multiple user groups

Hence it is important to enable the compatibility with each platform the application is supposed to run on. For the resulting n-to-n approach (figure 4) this can be implemented when a) the application is adapted to every single target platform b) uses an adequate virtual machine model, which is available for each target platform, or c) standards are employed, which can be used by every target platform.

Case A will deliver native applications to all registered user platforms and systems. This will require the applications to be compiled and adopted to every target platform supported by the provider. Similar deployment models can be seen for software as e.g. browsers, which are available for a whole set of operating systems (Mozilla, 2010). This is primarily the operating system. Possibly the caregivers' IS must also be considered if the application needs to be integrated with the main system on the end user. Without common communication interfaces, every version has to be adjusted manually. This model is known for mobile devices as well. Native software is adapted to each operating system as the iPhone OS, Android of Symbian.

Case B will require a virtual machine to run the source code on the target device. Such software implementations of computers are available for multiple platforms and can execute applications virtually. The Java Virtual Machine (JVM) is widely known and used. A similar model is feasible for medical IS when virtual machines are running on all target platforms. The interface problems can normally not be overcome, as only standard system interfaces are implemented in the virtual machine.

Case C: The Provider offers the developers applications via the standardized access, which is nearly universally executable by computers. The TCP/IP Stack can today be used with various application stacks, as the HTTP or SOAP protocol stack, on which distributed applications can be built. Therewith the applications are nearly universally 
accessible as internet browsers are broadly distributed, on personal computers (PC), smart phones and tablet devices. Software as a Service (SaaS) is the corresponding software delivery model, which provides access to business functionalities remotely (usually over the internet) as a service (Knorr, 2007). This approach can be integrated with existing legacy systems (Sun et al., 2007) as medical IS.

\subsection{Application Platforms for different groups of patients.}

We suggested some rough methods to approach the challenge of multiple target platforms and IS. A provider of an e-health marketplace can offer software with a certain standard or for a virtual machine to manage the fragmentation issue. The majority of healthcare spending is public funds. Those are credited to the caregiver after the treatment, mostly according to a fixed allowance catalogue. If the user of e-health applications is a caregiver, whose treatment is founded by his or her patient's health insurance, the software must be able to deal with the billing modalities of several user groups. Most insurance differ in terms of benefits and administration. In Germany each citizen has mandatory public health insurance. Currently 163 public health insurances are available (GKV, 2010), which leads to at least 163 different groups of clients to be handled.

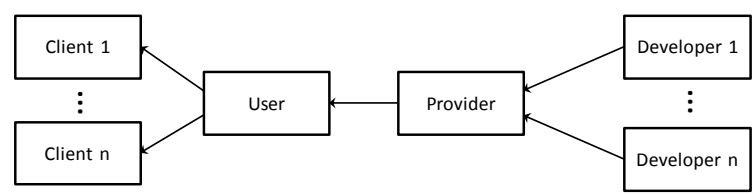

Figure 5: Application Platform with certification entity.

Clients insured by private institutions have different treatment conditions than clients who are insured by a public health insurance company. This can increase the diversity further. Considering the telemonitoring example for patients with cardiac insufficiency, the payment to patients, caregivers, and patients has to be included into the application. Each health insurance might pay different allowances to caregivers and patients according to their regulations. The provider of the application platform could offer the service of financial transactions as an additional feature if there is a link to the health insurance and a payment standard (figure 5).

\subsection{Application Platforms with certification entity}

Taking into account that health information contains very sensitive patient data, a single provider might not be suitable to control and host the applications in a self-contained way. The privacy concerns rose for HealthVault are likely to come up for other commercial providers that offer e-health applications, too. Compliance with legal requirements raises the necessity for a controlling entity, which is independent from the provider and separates commercial interests from those of the public health authorities and the patients. Applications might collect and interpret personalized medical data in an illegitimate way. The certification of a third party can eliminate such threats and increase the trust in e-health applications, especially if commercial interests of the controller can be disqualified (figure 6).

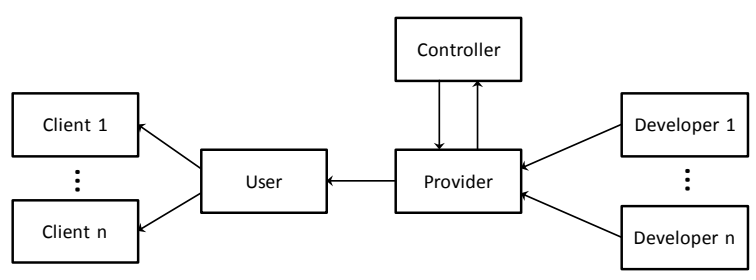

Figure 6: Application Platform with certification entity

The controlling entity is also suitable to set up regulations that any medical application has to obey. A reference model could be the HIPAA guideline or the German book of social law (Bundesrepublik Deutschland, 1988), which guarantees that e.g. every medical data item needs to be authorized by the owner, before it can be processes by a caregiver. Also the usage of encryption and digital signature are regulated in this legal framework.

\subsection{Multi User Application Platforms}

Customers buying e-health applications have to be distributed into at least two different groups. The telemonitoring example indicated that both, caregivers and patients, need software tools to ensure a proper monitoring of the patients' vital parameters. One group collects the data; the other group is responsible for the analysis. The application platform must therefore ensure access for patients and various groups of caregivers. The resulting model (figure 7) indicates that the provider has to offer to at least two access channels. 


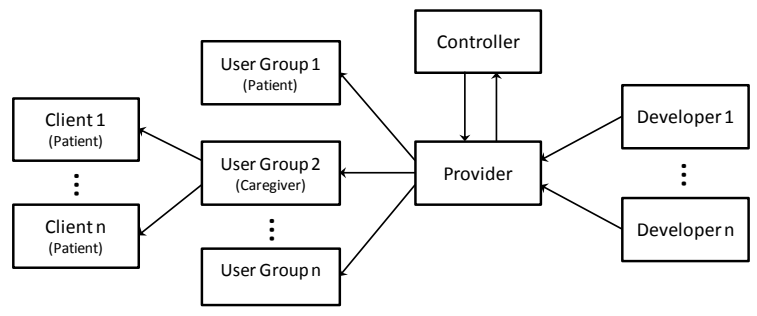

Figure 7: Multi User-Application Platform Service

The resulting model presents a comprehensive approach. It addresses all actors and requirements necessary to enable software-based cooperation between patients and caregivers. Legal compliance and an adequate security standard can be ensured by a controller entity. Developers of different nature can use the provider to offer their services to the users. Health insurances should be linked to the provider with a unified billing model. A payment for every transaction is more likely than a licence model that requires investment prior to the actual usage of the service. Each arrow in the model connects actors involved in the marketplace. All actors but the provider and the controller are currently very heterogeneous. A standardization of these links must therefore be pushed forward to avoid numerous adoptions to individual characteristics.

\section{CONCLUSIONS}

Construction of application stores for the healthcare domain is more complicated than for the mobile phone market. While mobile phone manufactures can rely on a unified target platform, the market for health IS is highly fragmented. Not only the diversity of end-user systems has to be targeted in an appropriate manner, but also security standards, the challenge of interoperability and the ability to bill services to multiple health insurances or authorities that operate with different treatment standards. A set of standards is essential to unify the system and organization landscape. Otherwise it is unlikely to achieve a workable platform, allowing more players to build up the market and pushing medical innovations into practise. International marketplaces are difficult to implement, as the health systems differ enormously around the globe. Already the number of requirements in the German public health system is huge. Theoretically 200 primary care systems are deployed; these have to interact with value added applications. Each application must be able to determine the treatment standard for at least 163 health insurances, which can result in very high complexity. Frequent changes in the legal regulations raise further barriers for a stable and sustainable operation of an ecosystem for e-health applications.

A marketplace for healthcare applications is certainly desirable. A standardized platform would ease the market access for more players and accelerate the diffusion of the innovations seen in the domain of e-health. Self-contained development of e-health is very difficult by today, as many requirements have to be converged to be fully compliant with national security and administrative standards. Authorities must set up adequate standards to enable software development for the healthcare market that is detached from the regulations and focuses on the medical and technical innovations, which are the core competence of the developers. Chapter 3.3 has indicated how the problems of platform diversity can be overcome in software deployment. Health insurances will have to develop similar methods to determine how e-health applications can be included into the regular billing model for healthcare services. A financial model focused only on the direct payment of patients is unlikely to be successful. Effectiveness of an application can often not be judged by the patients, an inclusion of caregivers seems therefore inevitable. Hence a proper incentive structure for this group is important. Health authorities can use the results of the studies to judge the effectiveness of e-health applications and include them as allowable services within a regular time cycle if they perform well. The paper worked out the actors involved in a potential marketplace and described important connections between them. The resulting model is a high level framework of a comprehensive platform for application distribution. A more detailed analysis of the technical and organizational requirements should be subject of further research.

\section{REFERENCES}

APPLEINSIDER (2010) Apple says App Store has made developers over $\$ 1$ billion.

ARANAZ-ANDRÉS, J. M., AIBAR-REMÓN, C. \& VITALLER-MURILLO, J. (2008) Incidence of adverse events related to health care in Spain: results of the Spanish National Study of Adverse Events. Journal of Epidemiology and Community Health, 62:, 1022-1029. 
BERNNAT, R. (2006) Kosten-Nutzen-Analyse der Einrichtung einer Telematik-Infrastruktur im deutschen Gesundheitswesen. Booz Allen Hamilton GmbH.

BUNDESREPUBLIK DEUTSCHLAND (1988) Sozialgesetzbuch (SGB) Fünftes Buch, Gesetzliche Krankenversicherung.

DÜNNEBEIL, S., SUNYAEV, A., BLOHM, I., LEIMEISTER, J. \& KRCMAR, H. (2010a) Do German Physicians want Electronic Health Services? A Characterization of Potential Adopters and Rejectors in German Ambulatory Care. Third International Conference on Health Informatics (HealthInf 2010). Valencia.

DÜNNEBEIL, S., SUNYAEV, A., LEIMEISTER, J. M. \& KRCMAR, H. (2010b) Strategies for development and adoption of EHR in German ambulatory care. 4th International Conference on Pervasive Computing Technologies for Healthcare (PervasiveHealth). Munich, IEEE.

EUROPEAN COMISSION (2007) EuropäischeUnion (2007) -eHealth for Safety Report. IN COMMUNITIES, O. F. O. P. O. T. E. (Ed.). Luxembourg.

GKV (2010) Alle gesetzlichen Krankenkassen. IN NATIONAL ASSOCIATION OF STATUTORY HEALTH INSURANCE PHYSICIANS (Ed.).

HELMS, T., PELLETER, J. \& RONNEBERGER, D. (2007) Telemedizinische Betreuung chronisch herzinsuffizienter Patienten am Beispiel des telemedizinischen Patientenbetreuungs- und schulungsprogramms ,Telemedizin fürs Herz". Herz, 32, 623-629.

HOLZER, A. \& ONDRUS, J. (2009) Trends in Mobile Application Development. Mobile Wireless Middleware, Operating Systems, and Applications - Workshops.

IOM (2006) Identifying and Preventing Medication Errors - Institute of Medicine,. IN INSTITUTE OF MEDICINE OF THE NATIONAL ACADEMIES (Ed.). Washington D.C., The National Academic Press.

KASSENÄRZTLICHE BUNDESVEREINIGUNG (2008) Grunddaten zur vertragsärztlichen Versorgung in Deutschland. Berlin.

KEMPF, K. \& SCHULZ, C. (2008) Telemedizin bei Diabetes: Höhere Therapiezufriedenheit, verbesserte Stoffwechselparameter. DiabetivaR Studie Monika Dienstle, WDGZ.

KNORR, E. (2007) Software as a Service: The Next Big Thing.

KURT, T. (2001) Großbritannien: Ärzte enttäuscht und desillusioniert. Deutsches Ärzteblatt, 98

KVB (2009) Installationsstatistik - Systeme. IN BUNDESVEREINIGUNG， K. (Ed.). Berlin.

LAPPÉ, J. M., MUHLESTEIN, J. B. \& LAPPÉ, D. L. (2004) Improvements in 1-Year Cardiovascular Clinical Outcomes Associated with a Hospital-Based Discharge Medication Program. Annals of Internal Medicine, 141, 446-453.

MICROSOFT (2007) Microsoft HealthVault Archtiecture Overview. Microsoft Corporation.

MOZILLA (2010) Download a Firefox version that speaks your language., The Mozilla Project.

PEDERSEN, S. \& HASSELBRING, W. (2004) Interoperabilität für Informationssysteme im Gesundheitswesen auf Basis medizinischer Standards. Informatik Forschung und Entwicklung, 18, 174-188.

ROGERS, E. M. (1995) Diffusion of innovations, New York, NY [u. a.], Free Press.

SUN, W., ZHANG, W., CHEN, S., ZHANG, X. \& LIANG, H. (2007) Software as a Service: An Integration Perspective. IN 4749, L. (Ed.) ICSOC 2007. Berlin Heidelberg, Springer-Verlag 2007.

SUNYAEV, A., CHORNYI, D., MAURO, C. \& KRCMAR, H. (2010) Evaluation Framework for Personal Health Records: Microsoft HealthVault vs. Google Health. Proceedings of the Hawaii International Conference on System Sciences (HICSS 43). Kauai, Hawaii.

TUFFS, A. (2008) Germany plans to introduce electronic health card BMJ.com Medical publication of the year.

ZUEHLKE, D., MEIXNER, G. \& KLEIN, U. (2010) Smart Medical Software Systems For Dummies? The Case For A UserCentered Systems Design. Third International Conference on Health Informatics (HealthInf 2010). Valencia. 